\title{
Improving the Accuracy, Adaptability, and Interpretability of SSD Failure Prediction Models
}

\author{
Chandranil Chakraborttii \\ University of California Santa Cruz \\ cchakrab@ucsc.edu
}

\begin{abstract}
Flash-based solid state drives represent an important storage tier in today's hyperscale data centers. Although solid state drives (SSDs) are relatively reliable, data center operators are interested in predicting future drive failures to administer drive replacement, data migration, and drive acquisition strategies. We analyze telemetry data from over 30,000 SSDs running live applications in Google's datacenters over a span of six years, for predicting and explaining SSD failures using machine learning techniques. We propose the use of 1-class isolation forest and autoencoder-based anomaly detection techniques for predicting previously unseen SSD failure types with high accuracy. We show that ignoring the minority class for training can improve the performance by up to $9.5 \%$ and if adaptability to dynamic environments is required, by up to $13 \%$. Furthermore, this paper proposes to utilize 1-class autoencoders to enable model interpretability. In particular, our autoencoder-based approach enables reasoning about the causes that lead to SSD failures. Common to all approaches, we deploy a set of powerful feature selection techniques that improve the model performance by up to $1.3 \times$ and reduce training times by up to $1.8 \times$.
\end{abstract}

ACM Reference Format:

Chandranil Chakraborttii and Heiner Litz . 2020. Improving the Accuracy, Adaptability, and Interpretability of SSD Failure Prediction Models. In Symposium on Cloud Computing (SoCC '20), October 19-21, 2020, Virtual Event, USA. ACM, New York, NY, USA, 14 pages. https://doi.org/10.1145/3419111.3421300

\section{INTRODUCTION}

NAND flash based solid state drives (SSDs) represent an important storage tier in data centers holding most of today's warm and hot data. Although SSDs are less cost-efficient than hard drives (HDDs), they provide several advantages

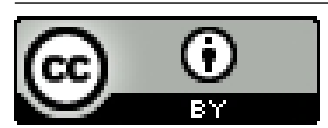

This work is licensed under a Creative Commons Attribution International 4.0 License.

SoCC '20, October 19-21, 2020, Virtual Event, USA

(C) 2020 Copyright held by the owner/author(s).

ACM ISBN 978-1-4503-8137-6/20/10.

https://doi.org/10.1145/3419111.3421300

\author{
Heiner Litz \\ University of California Santa Cruz \\ hlitz@ucsc.edu
}

over disks including orders of magnitude higher bandwidth (a million I/O operations per second), lower average read latency (less than $100 \mu \mathrm{s}$ ), and lower energy consumption. As SSDs are built from semiconductors lacking mechanical components such as spinning disks, they are also more reliable and less prone to failures compared to HDDs. The number of SSDs shipped each year has increased steadily by $42.5 \%$ over the last decade [1], now exceeding exabytes of storage capacity every year. SSD manufacturers have employed three main techniques to increase the storage density over the past years including planar scaling, 3D integration, and multi-level cells. While beneficial for the storage density, these mechanisms have reduced the endurance, retention, and reliability of SSDs [52], [13], [76], requiring increasingly sophisticated encoding and fault tolerance mechanisms. Nevertheless, even with advanced fault tolerance techniques and low failure rates, large Hyperscale data centers utilizing 100,000 's of SSDs suffer from multiple device failures daily. Data center operators are interested in predicting SSD device failures for two main reasons. First, even with RAID [6] and replication [25] techniques in place, device failures induce transient recovery and repair overheads affecting the cost and tail latency of storage systems. Second, predicting nearterm failure trends helps to inform the device acquisition process enabling to save costs and avoid capacity bottlenecks. As a result, it is important to predict both the short-term individual device failures as well as near-term failure trends.

Prior studies on predicting storage device failures [62], [53], [10], [75] focused primarily on traditional hard disks, however, due to the fundamentally different architecture of SSDs, prior techniques and findings are not readily applicable to SSDs. Research that has particularly focused on SSDs [8], [14], [19], [37] generally concentrated on understanding specific errors and issues within SSDs, limited to a controlled laboratory environment. Most studies that analyzed SSDs in the field focused on understanding correlations among specific workloads, their induced number of writes and bit errors, as well as their effect on the reliability of SSDs [21] [68], [23]. Alter [5] and Schroeder [69] analyzed authentic SSD logs collected in the Google cloud to leverage machine learning (ML) techniques for predicting the likelihood of SSD failures. While most related to our work, their proposed 
models either fall short on determining failed drives, or produce a large number of false positives, thereby lowering the performance of the prediction models. In particular, these two prior works suffer from the following main challenges. First, as they utilize black-box ML techniques, they are unaware of the underlying failure reasons rendering it difficult to determine the failure types that these models can predict. Second, the models in prior work struggle with dynamic environments that suffer from previously unseen failures that have not been included in the training set. These two challenges are especially relevant for the SSD failure detection problem which suffers from a high class imbalance. In particular, the number of healthy drive observations is generally orders of magnitude larger than the number of failed drive observations, thus posing a problem for many ML models.

To address these challenges, we propose to utilize 1-class $M L$ models that are trained only on the majority class. By ignoring the minority class for training, our 1-class models avoid overfitting to an incomplete set of failure types, thereby improving the overall prediction performance by up to $9.5 \%$ in terms of ROC AUC score. The benefit of our proposed technique becomes even more evident when we reduce the types of failures included in the training set of the baselines approaches, showing $13 \%$ to $33 \%$ improvements using our proposed 1-class approaches over prior work. Furthermore, we introduce a new learning technique for SSD failure detection, 1-class autoencoder, which enables interpretability of the trained models while providing high prediction accuracy. In particular, 1-class autoencoders provide insights into what features and their combinations are most relevant to flagging a particular type of device failure. This enables categorization of failed drives based on their failure type, thus informing about specific procedures (e.g., repair, swap, etc.) that need be applied to resolve the failure.

For analysis and evaluation of our proposed techniques, we leverage a cloud-scale dataset from Google that has already been used in prior work $[5,70]$. This dataset contains 40 million observations from over 30,000 drives over a period of six years. For each observation, the dataset contains 21 different SSD telemetry parameters including SMART (Self-Monitoring, Analysis and Reporting Technology) parameters, the amount of read and written data, error codes, as well as the information about blocks that became nonoperational over time. We determine the best performing ML approaches for predicting SSD failures and then explore optimization techniques, including feature selection and data normalization, to address the challenges of large feature spaces and highly imbalanced datasets. With these optimizations in place, our best approach outperforms all prior approaches by at least $9.5 \%$ ROC AUC score.

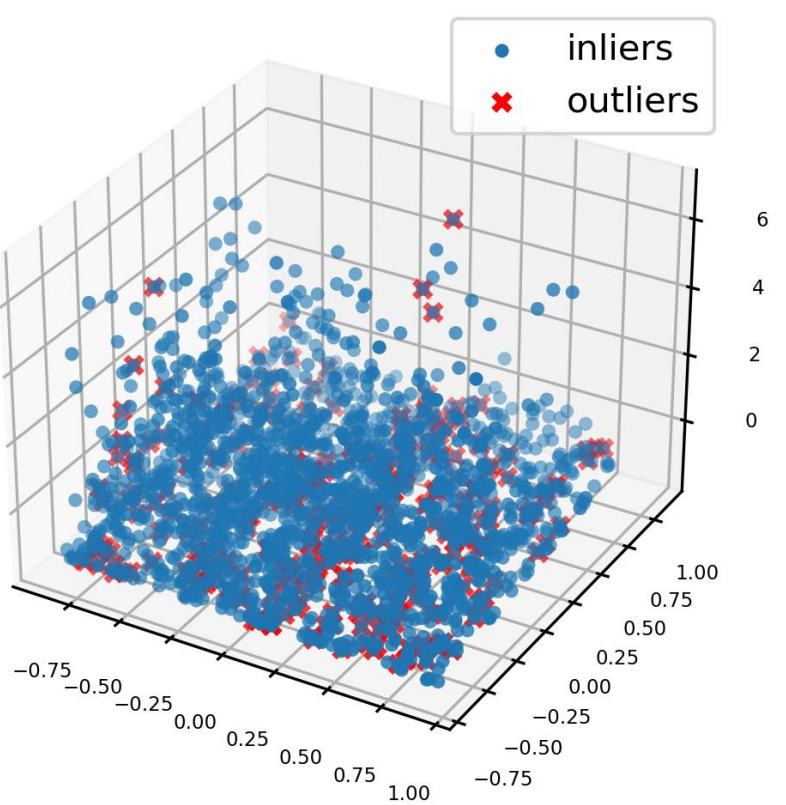

Figure 1: PCA with all 21 Features

\section{BACKGROUND}

This section provides a brief introduction to flash device technology and its implication on the reliability of SSDs. We show that predicting device failures is a challenging, multi-dimensional data problem that requires development of sophisticated machine learning techniques.

Contemporary SSDs are semiconductor devices that persistently store the data in NAND flash arrays consisting of floating gate transistors [38]. The charge within the floating gate can only be altered by applying a high voltage (20V), forcing the electrons to tunnel [28] through a highly resistive isolation material, thereby slowing degrading the storage capability and causing transistor wear-out. Furthermore, modern NAND drives leverage different voltage levels for storing multiple bits of information in a single transistor. Sensing the correct cell value on a read becomes increasingly difficult after writing the cell frequently, gracefully degrading the ability to read out data successfully. Unfortunately, due to manufacturing and device variability, the number of writes required to trigger a device failure is difficult to predict. As manufacturers want to increase their yields, many sold SSDs already contain a number of non-functioning bad blocks. Hence, the number of healthy blocks even differs among new devices, thus affecting their overall lifetime. The garbage collection and wear-levelling procedures performed by SSDs, internally, further impede the ability of detecting device failures. These tasks introduce extra writes, transparent to the user, which are highly application-specific. In 
particular, the write patterns (random vs. sequential) and read-write ratios affect the lifetime of a device considerably. In summary, the reasons leading to a particular device failure are highly application and device specific, rendering failure prediction by using a simple universal technique impossible. This is further motivated by Figure 1 showing a principle component analysis (PCA) for the 21 device-internal SSD features utilized in this study. As can be seen in this figure, several of the failed drives (outliers) cannot be easily separated from the the healthy drives (inliers), motivating the need for more sophisticated machine learning techniques.

\section{1-CLASS FAILURE PREDICTION}

Prior work on SSD failure prediction suffers from three shortcomings: (i) the limited overall accuracy of predicting failures, (ii) the inability of reliably predicting previously unseen failure types, and (iii) the lack of interpretability of predictions. To address these challenges, we provide the following contributions. First, we provide a comprehensive analysis of machine learning techniques to predict SSD failures with the highest recall and accuracy for both the majority and minority classes. We optimize our approaches by addressing the challenges of imbalanced data sets and feature explosion. Second, we show how 1-class predictive models can be used to predict previously unseen failures in a dynamic data center environment. Third, we propose 1-class autoencoder, an approach to interpret the predictions of our model, to enable understanding of the most important reasons for failures.

\subsection{Accurate Prediction of SSD Failures}

Our dataset contains 40 million observations from over 30,000 drives from Google data centers covering a time span of six years where each SSD observation contains the values of 21 distinct features. These features include SMART data, the amount of data read and written from the device, the emitted error codes, as well as the information about grown bad blocks which became non-operational over time due to wear-out. Predicting device failures from this data poses two challenging problems. First, due to the large feature space, machine learning models suffer from the curse of dimensionality, as the training and inference times of machine learning algorithms grow, often exponentially, with the number of features. Secondly, the data from which our models need to infer failures, suffers from a significant class imbalance problem, as there exists a significantly greater number of healthy drive observations than failed drive observations.

3.1.1 Feature Selection. To address the curse of dimensionality introduced by large number of feature, we developed a feature selection mechanism [22, 39, 40, 43] for improving SSD failure prediction. The goal is to select the smallest number of distinguishing features from this dataset to enable the highest accuracy and recall for detecting SSD failures. In contrast to prior work on finding anomalous behavior in cloud systems [79], we performed an extensive study of eight different filtering mechanisms to rank different features in the order of their importance for failure prediction. We observed that, except for the top 9 features selected, the order of the importance of features computed by the different feature selection algorithms varied substantially and, in fact, utilizing any one feature selection mechanism individually can lead to high variation in model performance. To address this challenge, we developed an approach to effectively combine the rankings of different feature selection algorithms, subsequently leading to the best model performance both in terms of training time and accuracy, as shown in Section 5.

3.1.2 Class Imbalance. A major challenge in anomaly detection is to deal with the inherent class imbalance problem. Among the over 30,000 drives that we examined, about 4,000 SSDs failed at some point in time, however, for the most of its lifetime, every SSD behaves like a healthy drive. This resulted in a training dataset containing over 40 million data points for healthy drives (majority class) while only 15,000 data points for failed drives (minority class). Distinguishing between healthy and failed drive observations is further aggravated by the fact that some of the drives were put back into service after repair and then failed again, requiring to be treated as separate failure observations.

Since the size of the majority class is three orders of magnitude larger than the size of the minority class, recognizing instances of the minority class during classification is challenging, since many of the ML algorithms are designed to be biased toward the majority class. The data points at which the minority instances are positioned among the majority instances in an imbalanced scheme contributes to the increase in misclassification rate, thus commonly referred to as data difficult factors [18]. These factors include, but are not limited to, small disjuncts, class overlap, borderline, noise, outliers, and rare instances [27].

Prior research [5], [79], [32], [54] used techniques including Random Forest [5, 46], Neural Networks [34], k-Nearest Neighbours (k-NN) [47], and 2-Class Support Vector Machines (SVM) [9] for predicting storage device failures. We observe that these approaches cannot cope well with the high class-imbalance and overfit to the failure types contained in the training dataset. In Section 5 we show that our proposed 1-class predictive models outperform prior works by up to $9.5 \%$ and reduce training times by up to $1.8 \times$. We also evaluate our proposed feature selection techniques and perform a sensitivity study on how far ahead the models can predict the failures. 


\subsection{Predicting unseen failures}

As outlined in Section 2, flash devices suffer from a variety of different failures induced by write amplification, grown bad blocks, controller errors, and backup battery issues. As some of the failures are workload-dependent and SSD technologies change, that is, the move from TLC to QLC cells, it is difficult to collect data about every failure type. Hence, it is unlikely that any training dataset would cover all types of device failures that may occur in the future.

We observed that previous approaches to detect SSD failures generally fail to predict unseen failure types that have not been experienced by the model during training. In this work, we propose to improve the adaptivity of the predictive models by training them only on the majority class instances. By utilizing only healthy drives as training data, the models can learn a strong representation of healthy drives, without overfitting to a limited set of known or previously seen failure types.

We introduce two mechanisms to enable this approach including 1-class isolation forest and 1-class autoencoders [73]. The generic isolation forest [50] is a popular algorithm for performing anomaly detection based on Random Forest. The algorithm leverages the fact that anomalous data points generally satisfy fewer conditions than normal data points. Hence, an anomaly score can be computed by counting whether the number of conditions required to separate a given data point is below a certain threshold. We also explored different contamination factors (the fraction of anomalous data points) to inform the model about this additional information. Utilizing these optimizations, we show in Section 5 that anomalous drives can be determined with a high recall of 0.99 , even though the model had never seen a failed drive during training.

Furthermore, to the best of our knowledge, this is the first work to use 1-class autoencoders for predicting SSD failures. We designed an 1-class autoencoder based model that generates a compressed knowledge representation of the original input of healthy drive observations as well as a trained decoder which, in return, tries to generate healthy drive observations from the compressed representation. We remove all failed drive observations from the dataset for training the autoencoder model, in order to enable the model to learn a compressed representation of what a healthy SSD should look like. Reconstruction error [66] is used to interpret the decisions emitted by this model. Figure 2 shows the internal design of autoencoders. We first encode and then decode a particular sample of SSD using the autoencoder model. If the input and output are similar, the input likely corresponds to a healthy drive, whereas, if the input and output suffer from a large reconstruction error, then the sample is flagged as an anomaly (failed drive).

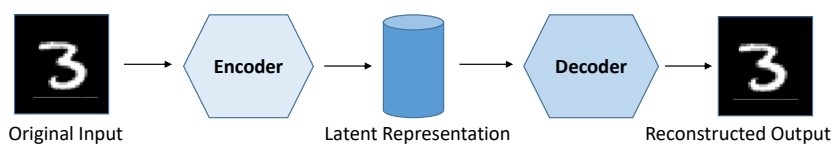

Figure 2: Autoencoder Design

As we show in Section 5, training on only the healthy drives provides the following benefits. First, the training is not limited by learning from a few samples in the minority classes. Second, the training examples from healthy drives are easier and cheaper to record, which improves the scalability of our approach. Third, ignoring the minority class during training improves the ability of the model to predict previously unseen failures.

\subsection{Interpreting SSD Failures}

Understanding the reasons for an SSD drive failure is of primary concern for manufacturers and data center operators to improve the reliability and to inform about the required maintenance and repair procedures. This enables them to choose appropriate drives for a particular workload, providing the best reliability as well as enabling fast re-servicing of drives. Providing an understanding of SSD failures also helps with increasing the transparency of our predictions and avoids running full diagnostic tests to determine the causes of a failure.

We leverage our neural network based 1-class autoencoder approach to enable this capability by creating a compressed lower-dimensional representation of healthy drive observations as explained in the previous section. We then use this representation to select anomalous observations that do not conform to the representation, thereby generating an output that differs significantly from representation of healthy observations. The observations that produce a reconstruction error greater than a chosen threshold are flagged as failures. We then categorize these generated outputs by separating them into buckets, each one representing the error while reconstructing the input for each feature. The features that produce a larger than average error for a particular drive are then marked as significant and reported. We show in Section 5 how interpreting this data provides insights into why the model predicted a particular device as a failed drive.

\section{METHODOLOGY}

Our dataset contains SSD Telemetry data from over 30,000 drives over a period of 6 years collected from Google datacenters. In total the dataset contains 40 million observations with 21 different telemetry parameters. Around 4,000 drives failed during this period leading to 15,000 observations classified as failed from the total of 40 million observations. 
The dataset contained information on four different SSD models (MLC A, B, C and D) and contained no information on specific vendors. Our feature selection process (see Section 5.4.2) did not select the model as a significant feature and hence we excluded it during training process. Of the drives that failed, approximately $90 \%$ of the drives failed only once while the rest failed up to four times. For our work, we label each failure as a separate case. Drive replacement times, upon failure varied widely ranging from under a week $(80 \%$ of the cases), to over three months ( $10 \%$ of the cases).

Around $30 \%$ of the drives that failed during the data collection process were replaced while the rest were removed, and hence no longer appeared in the dataset. As a result, we obtained approximately 300 observations for each healthy drive and 4 to 140 observations for each failed drive. The entire list of metrics of features present in the dataset is shown in Table 1.

Traditionally, the drive replacement policy at cloud service providers uses a rule-based approach [31]. Whenever certain parameters such as UECC error count, reserve block count, etc., reach a certain value, the drive is replaced. However, this approach suffers from two shortcomings. First, these rules do not comprehensively predict all the failures, and hence the drives fail unexpectedly in certain cases, resulting in data loss and application crashes. Second, these rule sets have also been shown to be overly conservative, leading to many cases where drives are replaced even though they were still operating normally. The aggregate number of drive failures per week is also beneficial for cloud providers as they can order replacements in advance. These issues motivated us to develop a more flexible and accurate approach based on machine learning techniques.

\subsection{Data Preprocessing}

The data collected contained features in string, date time, and integer format. We ensured that all the data collected was transformed into numeric format so that it can be processed by the machine learning models. String values, such as Drive model name, were converted into categorical features, and date and time were converted into UNIX timestamps. We treated each data point as an independent observation and normalized all the non-categorical data values to be between 0 and 1 . We created separate datasets, identified by the parameter $N$, by selecting daily observations before a predicted failure occurred. For instance, $N=3$ contains all observations for each drive 3 days before the drive either failed or was still functional. We leverage this data in order to find out how far ahead our proposed models can predict the failures.

\subsection{Feature Selection}

One of our primary goals was to select the most distinguishing features that are highly correlated to the failures for training. We used three different feature selection methods, Filter [67], Embedded [45], and Wrapper [71] techniques, and implemented eight different algorithms including Pearson ranking [60], Spearman ranking [77], Chi square test [56], Analysis of Variance (ANOVA) [35], Recursive Feature Elimination [29], Extra Trees [51], Lasso Regularization [81], Elastic Net [82], and Ridge Regression [30], for selecting the most important features contributing to failures for our dataset.

4.2.1 Filter Methods. Filter methods for feature selection use statistical measures to provide scores for each feature. The features were then ranked by this score and only the top significantly correlated features were selected. Specifically, we used Pearson correlation, Spearman correlation, Elastic Net, and Kendall Tau ranking algorithms to rank the features.

4.2.2 Wrapper Methods. Wrapper methods select different combinations of features and then evaluate them to pick the most relevant features. A prediction model is typically used to evaluate the combinations and assign scores based on model accuracy. We used different search processes including Random Forest, Recursive Feature Elimination with Extra Trees classifiers and Logistic Regression to select the top features.

4.2.3 Embedded Methods. Embedded methods pick the most relevant features that contribute to the accuracy of the model during the creation and training of the model. LASSO (L1), Elastic Net, and Ridge Regression (L2) are the most commonly used regularization methods. These methods optimize the learning procedure by training models with lower complexity, where features with non-zero coefficients are selected for training the model, thus serving as methods for feature selection. The three methods above provide feature rankings which were then merged into a single list, giving equal importance to each method. As we show in Section 5, the elaborate feature selection process improves both the training time and the prediction accuracy significantly over the baseline that utilizes all 21 features. The resulting set of top features is shown in Table 2. We validated the feature selected with domain experts, who confirmed that there is a strong correlation between the features that were picked by the feature selection algorithms and actual parameters which indicate wear out and failures in SSDs.

\subsection{1-Class ML Models}

For training the 1-class models, autoencoder and isolation forest, we used the $\mathrm{H} 2 \mathrm{O}$ library [20] and split the dataset into training and test set. The training set contains data from 


\begin{tabular}{|l|l|l|}
\hline Features & Datatype & Description \\
\hline \hline drive id & string & Unique ID assigned to each drive \\
\hline model & string & Drive model type \\
\hline timestamp & int & Time (in us) since the drive was first put in use \\
\hline read count & int & Number of read operations in the drive's lifetime \\
\hline write count & int & Number of write operations in the drive's lifetime \\
\hline erase count & int & Number of erase operations in the drive's lifetime \\
\hline status read only & boolean & Status flag indicating if the drive is operating in read only mode \\
\hline cumulative p/e cycle & int & Number of times a memory cell is erased and reprogrammed \\
\hline factory bad block count & int & Number of non-operational data blocks upon drive purchase \\
\hline cumulative bad block count & int & Number of blocks which became non-operational during the drive's lifetime \\
\hline status dead & boolean & Status flag indicating if the drive is currently failed \\
\hline correctable error count & int & Number of uncorrectable ECC errors during read \\
\hline erase error & int & Number of erase operations that resulted in an error \\
\hline final read error & int & Number of read operations that resulted in an error, even upon retry \\
\hline final write error & int & Number of write operations that resulted in an error, even upon retry \\
\hline meta error & int & Number of errors while accessing the drive's internal metadata \\
\hline read errror & int & Number of read operations that resulted in error, but succeeded upon retry \\
\hline response error & int & Number of bad responses from the drive \\
\hline timeout error & int & Number of operations that timed out without completion \\
\hline uncorrectable error (UECC) & int & Number of uncorrectable ECC errors encountered during read operations \\
\hline write error & int & Number of write operations that resulted in error, but succeeded upon retry \\
\hline
\end{tabular}

Table 1: All 21 features collected

\begin{tabular}{|l|}
\hline Final Selected Top Features \\
\hline \hline correctable error count \\
\hline cumulative bad block count \\
\hline cumulative p/e cycle \\
\hline erase count \\
\hline final read error \\
\hline read count \\
\hline factory bad block count \\
\hline write count \\
\hline status read only \\
\hline
\end{tabular}

Table 2: Top features selected

$90 \%$ of the healthy drives but does not contain any samples of failed drives. For 1-class isolation forest, we use 250 trees, with a max_depth of 20 to get a good representation of a healthy drive from the input data. Increasing the tree size and max_depth beyond these values decreased precision of the model, indicating overfitting. We also experimentally explored the best value for the contamination factor hyperparameter. The initial hyperparameter values were based on domain knowledge and we performed extensive parameter sweeping and tuning (also for the baselines) to come up with the final hyperparameter values and models. While our training set has zero contamination (no failed drives), we need to inform the model about the contamination factor during inference so that the model can adjust the threshold to select between failed and healthy drives. The empirically determined contamination factor depends on the number of days the model needs to predict ahead and ranges between 0.016 and 0.002 .

The 1-class autoencoder model utilizes 4 hidden layers comprising of 50, 25, 25 and 50 neurons respectively. The neurons utilize a tanh activation function. We utilize the Adam optimizer [80] and train the model for 100 epochs. We use early stopping with a patience value of 5 ensuring that the training of the model stops when the loss does not decrease after 5 consecutive epochs. Increasing the number of hidden layers beyond 4 increases the training time significantly without providing performance benefits. We use 10 -fold cross validation to evaluate all models.

\subsection{Deployed System}

The processed dataset containing only the top selected features is subsequently used for training the different ML models. In a datacenter we envision our SSD failure prediction technique to be implemented as shown in the block diagram in Figure 3. The telemetry traces are collected periodically from all SSDs in the datacenter and sent to the preprocessing pipeline transforming all input data into numeric values while filtering out incomplete and noisy values. Following data preprocessing, feature selection is performed to extract 
the most important features from the data set. The preprocessed data is then either utilized for training or inference. For inference, device anomalies are reported and classified according to our 1-class autoencoder approach. SSDs can then be manually analyzed by a technician or replaced directly. As an alternative, a scrubber can be leveraged to validate the model predictions by performing a low level analysis of the $\mathrm{SSD}$, finding grown bad sectors and other drive issues.

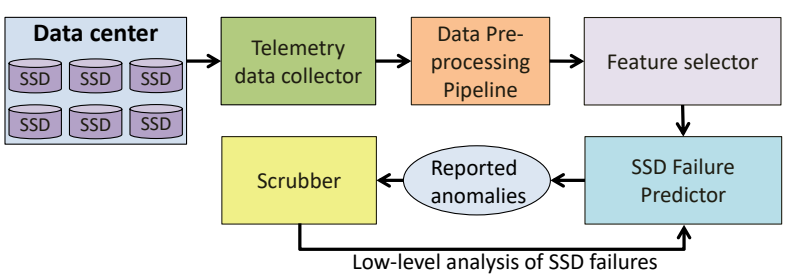

Figure 3: Block diagram of the Deployed System

\section{RESULTS}

In this section, we compare the performance of our proposed 1-class isolation forest and 1-class AutoEncoder techniques to three baselines used in prior work. In particular, we compare against, Random Forest, 2-Class SVM, and Neural Networks $(\mathrm{NN})$ as those have been used in prior work on SSD failure detection [5]. For the baselines, whenever available, we use the same model architecture and hyperparameters as proposed in prior work [5]. For the hyperparameters that we could not find in prior work, we performed a design space exploration and report the best numbers that we could find.

For SSD failure prediction, the primary goal is to predict all SSD failures since the cost of not capturing (mispredicting) a drive that is going to fail is higher than classifying a healthy drive as a failure which can be refuted by scrubbing [55]. Nevertheless, as performing scrubbing induces a performance overhead, achieving both high recall for failed devices and high accuracy for healthy devices is important as well. To satisfy these requirements, for all the experiments, we chose a high enough threshold to capture failures with a minimum recall value of 0.99 and then try to predict these failures with the fewest number of false positives.

For imbalanced datasets, traditional metrics (accuracy, precision, recall and fscore) alone can be deficient in measuring the performance of the classifier. Since the dataset is imbalanced, overfitting to the majority class (predicting all observations as the majority class) can skew performance and still reflect good overall precision, recall and fscore. The receiver operating characteristic curve, or ROC curve [11], is a graphical plot that illustrates the diagnostic ability of a binary classifier system as its discrimination threshold is varied. The ROC curve is created by plotting the true positive rate (TPR) against the false positive rate (FPR) at various threshold values. The true-positive rate is also known as sensitivity, recall or probability of detection in machine learning [65]. The false-positive rate is also known as probability of false alarm [24] and can be calculated as (1 - specificity). The area under the curve (ROC AUC) [26] is calculated to give a single score for a classifier model across all threshold values. This is inline with prior work that utilizes the ROC AUC metric for evaluating anomaly detection models [5].

To evaluate the five ML techniques we first label all 40 million observations in the dataset to separate between healthy and failed drive observations. We then perform a $90 \%-10 \%$ split of the dataset into training set and evaluation set. For training the 1-class models we remove all failed drive observations from the training set, however, the evaluation set is identical between our proposed 1-class techniques and the three baselines. We use 10 -fold cross validation for evaluating all approaches.

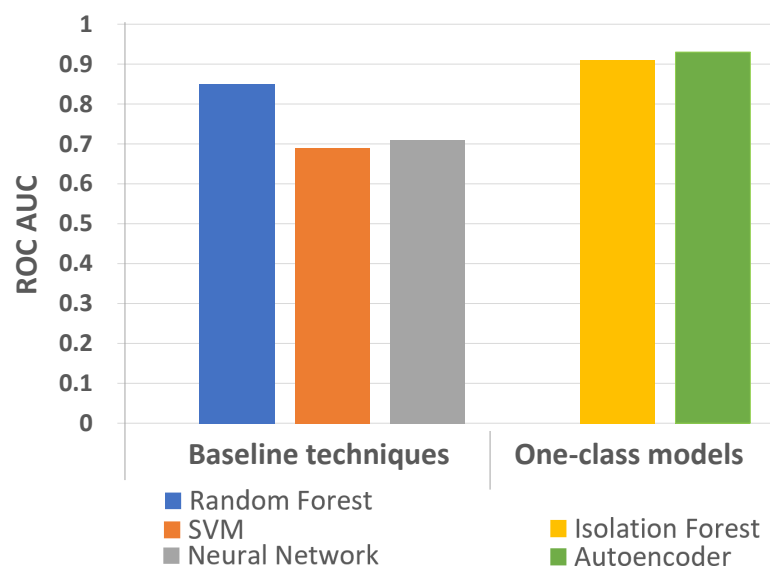

Figure 4: ROC AUC Score comparison of the five evaluated Machine Learning Techniques

\subsection{Accurate Prediction of SSD Failures}

Figure 4 illustrates the comparative performance of different ML techniques for predicting SSD failures one day ahead. Among the baselines, Random Forest performs best, providing a ROC AUC score of 0.85 . Both our 1-class models outperform the best baseline. In particular, 1-class isolation forest achieves a ROC AUC score of 0.91, representing a 7\% improvement over the best baseline while 1-class AutoEncoder, outperforms Random Forest by $9.5 \%$.

ROC AUC determines the ability of a model to distinguish between classes (failed vs. healthy in our application). To achieve good performance, models need to achieve both high recall and precision for both failed and healthy classes. Figure 5 explores these metrics for the five approaches in more detail for $\mathrm{N}=1$. 


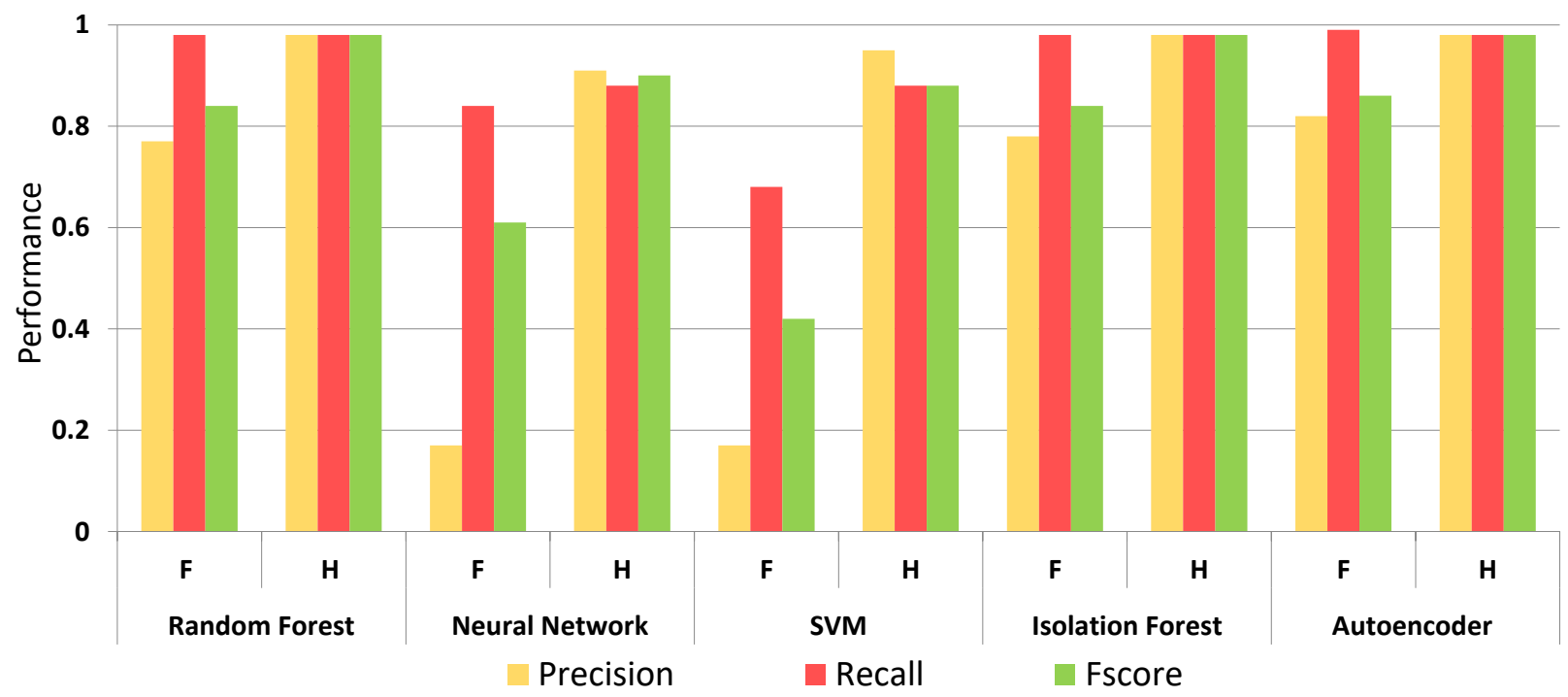

Figure 5: Accuracy, Precision, Recall and Fscore for the five evaluated Machine Learning Techniques

It shows that Random Forest performs equally well than our proposed 1-class Models in terms of Precision and Recall on the majority class of healthy $(\mathrm{H})$ drives, however, performs considerably worse on predicting the minority class of failed (F) drives. For the minority class, 1-class AutoEncoders improve precision by $6 \%$ over Random Forest as well as by $68 \%$ and $72 \%$ over the Neural Network and SVM baselines respectively.

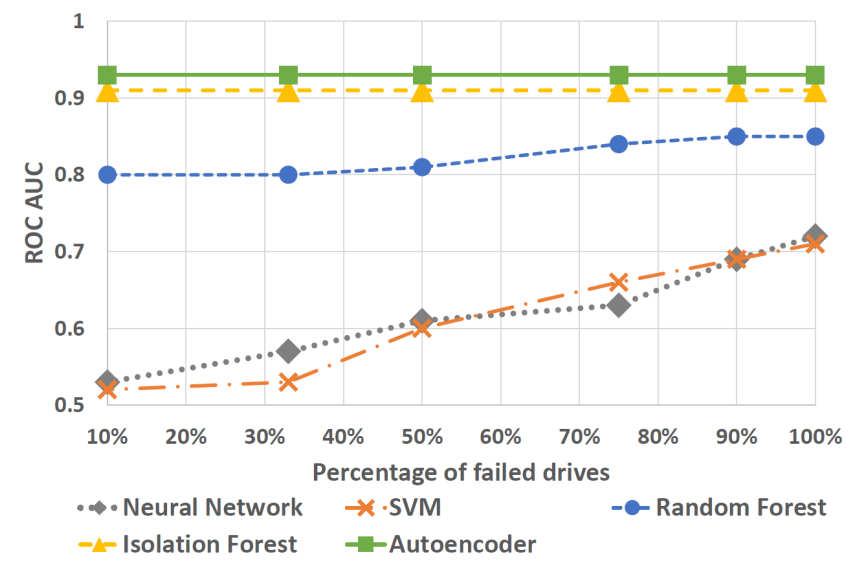

Figure 6: Predicting unseen failures

\subsection{Adaptivity to Unseen Failures}

In the previous section we showed that our proposed 1-class models are capable of outperforming the 2-class models by up to $72 \%$ under the best case scenario for the baselines in terms of precision (31\% in terms of ROC AUC score), where $90 \%$ of all failures types are contained in the training set. We now evaluate the model's ability in adapting to new datacenter environments, induced, for instance, by new workloads or new hardware. Therefore, in Figure 6, we sweep the number of failed drive observations included in the training set from $10 \%$ to $100 \%$, simulating dynamic environments where new failure types emerge over time.

Figure 6 shows the ROC AUC score for the three baselines and our proposed 1-class techniques with a variable percentage of failed drives included in the training set. Note that our 1-class techniques do not include any failed drives in the training set and hence we plot their performance as a straight line. The baselines' performance, however, depends significantly on the number of minority samples in the training set. For instance, if only $50 \%$ of the failed drive observations are included in the training set, our proposed 1-class Autoencoder technique outperforms Random Forest by $13 \%$ and NN and SVM by $33 \%$. This shows that particularly in dynamic environments, our 1-class techniques are a better choice than the techniques utilized in prior works.

\subsection{Interpreting SSD Failures}

This work proposes 1-class Autoencoders for interpreting SSD failures. In particular, our technique exposes the reasons determined by our model to flag a particular device failure. This is achieved by utilizing the reconstruction error generated by the model while reproducing the output using the trained representation of a healthy drive. The failed drives do not conform to the representation, thereby, generating 


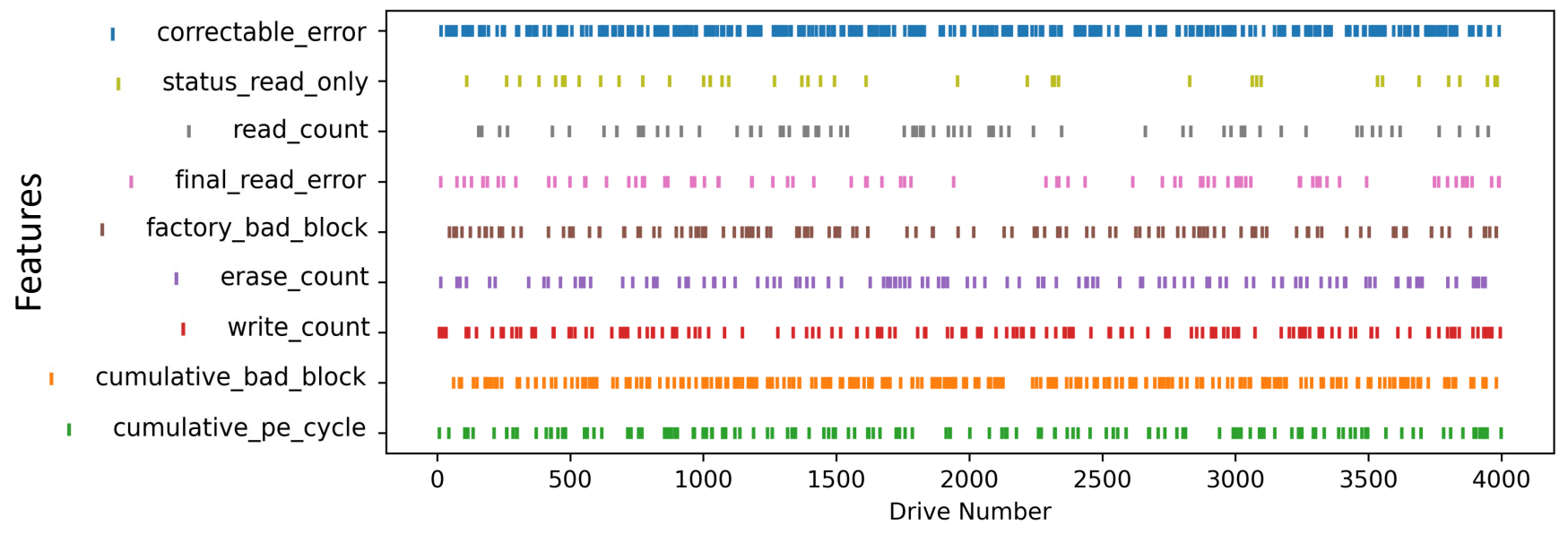

Figure 7: Interpreting SSD Failure Reasons

an output which differs significantly from the actual observations producing a large reconstruction error. We study the reconstruction error per feature to generate the failure reasons. The features which contribute more than the average error per feature to the reconstruction error, is defined as a significant reason.

Figure 7 shows how often a feature was flagged as a significant failure reason by the autoencoder model, aggregated for all observations from failed drives. The y-axis displays all features utilized by the model, representing a potential failure reason while the $\mathrm{x}$-axis shows the failed drive number. For each drive, we report the failure reason by means of a scatterplot. From Figure 7, we can see that many failed drives show a higher than normal number of correctable_errors counting the number of failed reads that could be corrected leveraging error correcting codes (ECC). This indicates that a high number of uncorrectable errors frequently leads to failures, however, it is also only a significant feature in approximately $35 \%$ of the drives.

Cumulative_bad_block represents another important reason determined by the model indicating SSD failures as it shows frequent anomalies, however, again only in less than $30 \%$ of the cases. In summary, this analysis shows that there exist particularly relevant features that indicate device failures in many cases, however, only the combination of several features enables accurate failure prediction. We also note that, cumulative UEC (Uncorrectable error count) which has been researched extensively $[15,36,64]$ for SSD failure correlation contributed to less than $1 \%$ of the failures according to our Autoencoder based model.

\subsection{Sensitivity Studies}

In the following we provide two additional sensitivity studies. In the first, we evaluate the ability of our models to predict failures multiple days in advance. Predicting further ahead is beneficial for logistical reasons and acquisition purposes. In the second study, we evaluate the effect of feature selection on the five approaches.

5.4.1 Predicting ahead in Time. To optimize drive maintenance and the acquisition of new spare drives, it is preferable to predict drive failures further ahead in time. While the previous sections have focused on predicting one day ahead, Figure 8 evaluated ROC AUC performance on predicting multiple days $(\mathrm{N})$ ahead. As expected, for all five models, prediction performance degrades when predicting further ahead. So while for 1-class Autoencoders performance degrades considerably, 1-class isolation forest can maintain the performance better. In particular, for $N=4$, the 1-class isolation forest model becomes the best performing technique outperforming the Random Forest baseline by $6 \%$ and SVM and NN by $11 \%$ and $13 \%$ correspondingly in terms of RUC-AUC score.

5.4.2 Feature Selection. As mentioned above we used feature selection algorithms for selecting the most important features contributing to failures in our dataset. Tables 1 and 2 list the features before and after feature selection. Figure 9 demonstrates the potential benefit of using feature selection by comparing the model performance (in terms of ROC AUC score improvement) with the original 21 features against the performance of the model trained with only 9 features. As can be seen, all techniques benefit from feature selection, for instance, Random Forest's absolute ROC AUC score improves by $3.7 \%$ when utilizing feature selection, while 1-class 


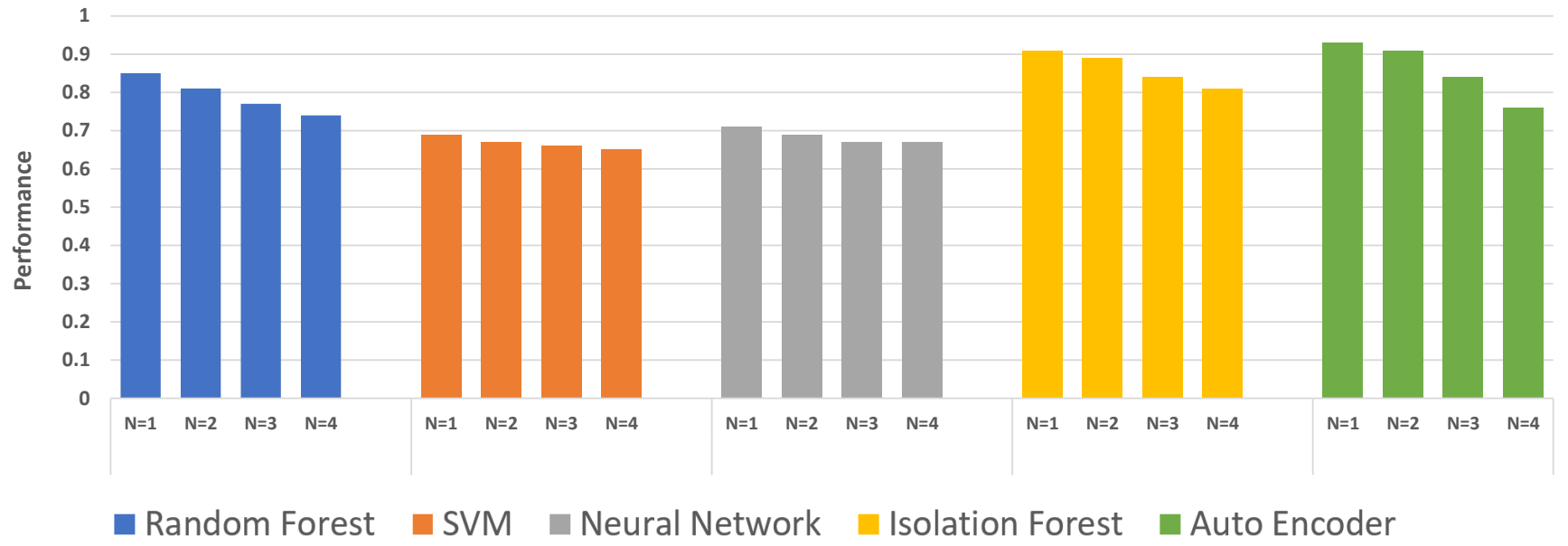

Figure 8: ROC AUC score when predicting up to 4 days ahead

Autoencoder's ROC AUC performance increases by $3.3 \%$. Feature selection also reduces the number of features used for training the models resulting in up to $41 \%$ (for autoencoder models) reduction in training times as can be seen from Table 3.

\section{DISCUSSION}

We discuss our proposed 1-class models below.

\subsection{1-Class Isolation Forest}

Anomaly detection approaches leveraging isolation forests are generally trained on both the majority and minority class. Perhaps surprisingly, we found that isolation forests trained only on the minority class performed exceptionally well, particularly for detecting unseen failures outperforming the performance of baseline approaches (Random Forest, 2 class SVM and Neural Network based models). 2-class models use data from both classes to learn a representation for each class. Since the number of samples for failed drives in our case is significantly lower than good working SSDs, the model has less samples to learn from and hence is more likely to misclassify previously unseen failed SSDs. 1-class models, in contrast, learn a representation of a good working SSD and are more likely to classify previously unseen anomalies correctly (1-class models do not suffer from overfitting to the limit training set of failed SSDs).

Our approach does not require training on all different failure types to detect failures and hence both generalizes and scales well when provided with new healthy observations. The approach outperforms autoencoders when predicting more than two days ahead and is faster to train requiring fewer training samples. Nevertheless, it was not able to outperform autoencoders (for $\mathrm{N}=1$ and $\mathrm{N}=2$ ) due to a higher false positive rate (anomalies as reported by the model which are not actual failures). We plan to use second level supervised binary classification in the future to teach the model about the known failures to eliminate more false positives during evaluation.

\subsection{1-Class Autoencoder}

To our knowledge, this is the first application of a deep learning based 1-class autoencoder for predicting SSD failures. We used the data from healthy drives to create an encoded representation of a healthy drive. Upon providing the test data

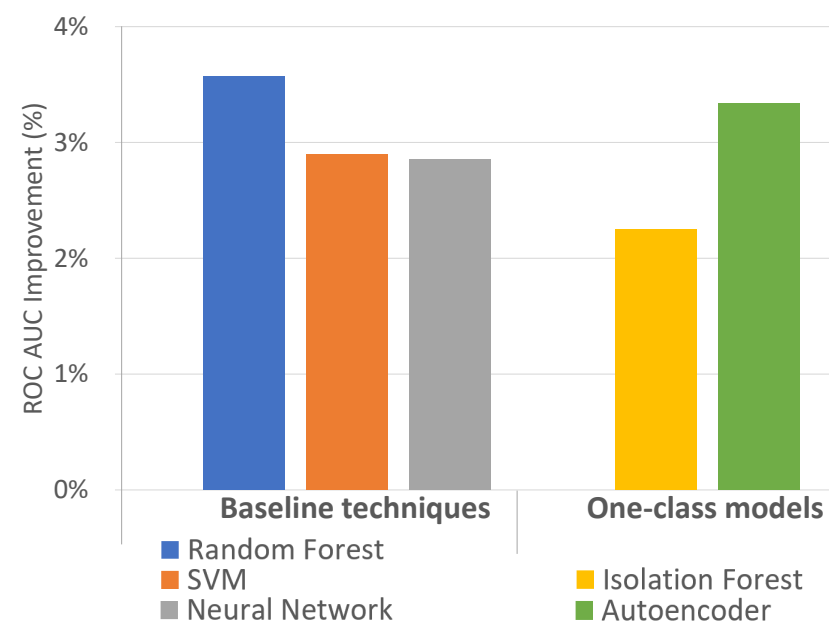

Figure 9: Impact of Feature Selection Techniques 
points to the encoded representation, we recorded the difference between the observed and generated output. Since the anomalous data points do not fit the encoding well, they tend to have higher error values. As in 1-class isolation forests, the autoencoder does not need to train on the minority dataset. autoencoders performed best while predicting failures up to 2 days ahead, achieving highest accuracy, precision and ROC AUC score with a recall of 0.99 . It performed worse than 1class isolation forests when predicting ahead 3 or more days achieving lower precision, however, autoencoders enable interpretation of the model predictions. In particular, we can learn why the model flagged an observation as a failure to inform the repair and maintenance procedure.

\begin{tabular}{|l|c|c|}
\hline ML Technique & Features & Training Time (sec) \\
\hline \hline \multirow{2}{*}{ Random Forest } & 9 & 695.4 \\
\cline { 2 - 3 } & 21 & 1095.6 \\
\hline \multirow{2}{*}{ Neural Network } & 9 & 1496.87 \\
\cline { 2 - 3 } & 21 & 2550.87 \\
\hline \multirow{2}{*}{ SVM } & 9 & 1156.6 \\
\cline { 2 - 3 } & 21 & 1885.6 \\
\hline \multirow{2}{*}{ Isolation forest } & 9 & 499,57 \\
\cline { 2 - 3 } & 21 & 686.54 \\
\hline \multirow{2}{*}{ Autoencoder } & 9 & 1750.57 \\
\cline { 2 - 3 } & 21 & 2781.89 \\
\hline
\end{tabular}

Table 3: Model training time $(\mathrm{N}=\mathbf{1})$

\section{RELATED WORK}

Earlier work on analyzing and predicting failures in storage systems focused on spinning disk drives [10, 32, 32, 53, 55, $59,62,68,75,78,78]$. However, due to the fundamentally different storage technologies these prior results are not applicable to flash based SSDs [17]. Furthermore, these prior studies were performed on a much smaller number of disk drives and hence were incompatible with ML techniques that require large training data sets.

Several studies have focused on providing statistics on long term failure trends [5, 10, 10, 52, 74]. Meza [57] has explored SSD failure prediction based on a single feature (uncorrectable bit-error rate) whereas our approach analyses a much more comprehensive set of 21 features. Schroeder [69] used supervised ML techniques (2-Class SVM and Random Forest) to predict sector failures. Alter [5] studied correlations between different workload conditions to study infantmortality of SSDs within Google's data centers. We contribute over these works by proposing 1-class models improving prediction accuracy, providing adaptivity to previously unseen failures, and enabling interpretability of predictions.
Anomaly detection techniques using both traditional machine learning [61] and deep learning techniques [63] have been successfully applied in various fields of research. Adewumi [2] provide a detailed review of deep learningbased techniques for fraud detection. A broad survey of deep anomaly detection (DAD) methods for cyber-intrusion detection is presented by Kwon [44]. An overview of DAD techniques for the Internet of Things (IoT) and big-data anomaly detection is introduced by Mohammadi and Mehdi [58]. Sensor networks anomaly detection has been reviewed by Ball [7]. The state-of-the-art deep learning based techniques for video anomaly detection along with various categories have been presented in Kiran [41]. Other applications of anomaly detection include predicting failures in cloud systems [72], the medical domain [48], and self-driving vehicles [4] [3]. Zhang [79] introduced ATAD, a method of detecting anomalies in cloud systems, by training the model on one dataset and using transfer learning to use the model for another dataset. Our work contrasts in using a combination of several feature selection techniques to select the most relevant features for training the model for generating failure reasons. In addition to anomaly detection applications, machine learning has been applied to improve the performance and efficiency of storage systems and SSDs [12, 16, 33, 42, 49].

\section{CONCLUSION}

This paper provides a comprehensive analysis of machine learning techniques to predict SSD failures in the cloud. Therefore, we collect SSD telemetry information from over 30,000 drives over a period of six years from Google's datacenters. We observe that prior works on SSD failure prediction suffers from the inability to predict previously unseen failure types motivating us to explore 1-class machine learning models such as 1-class isolation forest and 1-class autoencoder. We show that our approaches outperform prior work by $9.5 \%$ ROC-AUC score by significantly improving on the prediction accuracy for failed drives. For dynamic environments, where only a subset of the different drive failure types are part of the training set, our 1-class techniques improve over the baselines by $13 \%$. Finally, we show that 1 -class autoencoders enable interpretability of model predictions by exposing the reasons determined by the model for predicting a failure.

\section{ACKNOWLEDGEMENTS}

We thank Arif Merchant from Google for providing access to the Google traces and helpful feedback on the manuscript. We also thank the anonymous reviewers for their comments. This work was generously supported by Samsung Semiconductor Inc. and NSF grant \#1942754. 


\section{REFERENCES}

[1] [n.d.]. HDDs and SSDs: global shipments 2015-2021. https://www.statista.com/statistics/285474/hdds-and-ssds-inpcs-global-shipments-2012-2017/. Accessed: 2019-11-11.

[2] Aderemi O Adewumi and Andronicus A Akinyelu. 2017. A survey of machine-learning and nature-inspired based credit card fraud detection techniques. International fournal of System Assurance Engineering and Management 8, 2 (2017), 937-953.

[3] Khattab M Ali Alheeti, Anna Gruebler, Klaus D McDonald-Maier, and Anil Fernando. 2016. Prediction of DoS attacks in external communication for self-driving vehicles using a fuzzy petri net model. In 2016 IEEE International Conference on Consumer Electronics (ICCE). IEEE, 502-503.

[4] Khattab M Ali Alheeti, Anna Gruebler, and Klaus McDonald-Maier 2016. Intelligent intrusion detection of grey hole and rushing attacks in self-driving vehicular networks. Computers 5, 3 (2016), 16.

[5] Jacob Alter, Ji Xue, Alma Dimnaku, and Evgenia Smirni. 2019. SSD failures in the field: symptoms, causes, and prediction models. In Proceedings of the International Conference for High Performance Computing, Networking, Storage and Analysis. ACM, 75.

[6] Mahesh Balakrishnan, Asim Kadav, Vijayan Prabhakaran, and Dahlia Malkhi. 2010. Differential raid: Rethinking raid for ssd reliability. $A C M$ Transactions on Storage (TOS) 6, 2 (2010), 1-22.

[7] John E Ball, Derek T Anderson, and Chee Seng Chan. 2017. Comprehensive survey of deep learning in remote sensing: theories, tools, and challenges for the community. Journal of Applied Remote Sensing 11, 4 (2017), 042609.

[8] Hanmant P Belgal, Nick Righos, Ivan Kalastirsky, Jeff J Peterson, Robert Shiner, and Neal Mielke. 2002. A new reliability model for post-cycling charge retention of flash memories. In 2002 IEEE International Reliability Physics Symposium. Proceedings. 40th Annual (Cat. No. 02CH37320). IEEE, 7-20.

[9] Kristin P Bennett and Ayhan Demiriz. 1999. Semi-supervised support vector machines. In Advances in Neural Information processing systems. 368-374.

[10] Mirela Madalina Botezatu, Ioana Giurgiu, Jasmina Bogojeska, and Dorothea Wiesmann. 2016. Predicting disk replacement towards reliable data centers. In Proceedings of the 22nd ACM SIGKDD International Conference on Knowledge Discovery and Data Mining. ACM, 39-48.

[11] Andrew P. Bradley. 1997. The use of the area under the ROC curve in the evaluation of machine learning algorithms. Pattern Recognition (1997), 1145-1159.

[12] Peter Braun and Heiner Litz. 2019. Understanding Memory Access Patterns for Prefetching. In International Workshop on AI-assisted Design for Architecture (AIDArc), held in conjunction with ISCA.

[13] Yu Cai, Yixin Luo, Saugata Ghose, and Onur Mutlu. 2015. Read disturb errors in MLC NAND flash memory: Characterization, mitigation, and recovery. In 2015 45th Annual IEEE/IFIP International Conference on Dependable Systems and Networks. IEEE, 438-449.

[14] Yu Cai, Onur Mutlu, Erich F Haratsch, and Ken Mai. 2013. Program interference in MLC NAND flash memory: Characterization, modeling, and mitigation. In 2013 IEEE 31st International Conference on Computer Design (ICCD). IEEE, 123-130.

[15] Yu Cai, Yunxiang Wu, and Erich F Haratsch. 2016. Error correction code (ECC) selection using probability density functions of error correction capability in storage controllers with multiple error correction codes. US Patent 9,419,655.

[16] Chandranil Chakraborttii and Heiner Litz. 2020. Learning I/O Access Patterns to Improve Prefetching in SSDs. ICML-PKDD (2020).

[17] Chandranil Chakraborttii, Vikas Sinha, and Heiner Litz. [n.d.]. SSD QoS Improvements through Machine Learning.
[18] Nitesh V Chawla, Kevin W Bowyer, Lawrence O Hall, and W Philip Kegelmeyer. 2002. SMOTE: synthetic minority over-sampling technique. Journal of artificial intelligence research 16 (2002), 321-357.

[19] Tae-Sun Chung, Dong-Joo Park, Sangwon Park, Dong-Ho Lee, SangWon Lee, and Ha-Joo Song. 2009. A survey of flash translation layer. fournal of Systems Architecture 55, 5-6 (2009), 332-343.

[20] Darren Cook. 2016. Practical machine learning with H2O: powerful, scalable techniques for deep learning and AI. " O'Reilly Media, Inc.".

[21] Jim Cooke. 2007. The inconvenient truths of NAND flash memory. Flash Memory Summit 3, 3 (2007), 3-1.

[22] Manoranjan Dash and Huan Liu. 1997. Feature selection for classification. Intelligent data analysis 1, 1-4 (1997), 131-156.

[23] Robin Degraeve, F Schuler, Ben Kaczer, Martino Lorenzini, Dirk Wellekens, Paul Hendrickx, Michiel van Duuren, GJM Dormans, Jan Van Houdt, L Haspeslagh, et al. 2004. Analytical percolation model for predicting anomalous charge loss in flash memories. IEEE Transactions on Electron Devices 51, 9 (2004), 1392-1400.

[24] Jim D Echard. 1991. Estimation of radar detection and false alarm probability. IEEE Trans. Aerospace Electron. Systems 27, 2 (1991), 255260.

[25] Evangelos S Eleftheriou, Robert Haas, Xiaoyu Hu, and Roman A Pletka. 2014. Reliability scheme using hybrid SSD/HDD replication with log structured management. US Patent 8,700,949.

[26] Tom Fawcett. 2006. An introduction to ROC analysis. Pattern recognition letters 27, 8 (2006), 861-874.

[27] Alberto Fernández, Salvador Garcia, Francisco Herrera, and Nitesh V Chawla. 2018. SMOTE for learning from imbalanced data: progress and challenges, marking the 15-year anniversary. Journal of artificial intelligence research 61 (2018), 863-905.

[28] Ralph Howard Fowler and Lothar Nordheim. 1928. Electron emission in intense electric fields. Proceedings of the Royal Society of London. Series A, Containing Papers of a Mathematical and Physical Character 119, 781 (1928), 173-181.

[29] Pablo M Granitto, Cesare Furlanello, Franco Biasioli, and Flavia Gasperi. 2006. Recursive feature elimination with random forest for PTR-MS analysis of agroindustrial products. Chemometrics and Intelligent Laboratory Systems 83, 2 (2006), 83-90.

[30] Quanquan Gu, Zhenhui Li, and Jiawei Han. 2012. Generalized fisher score for feature selection. arXiv preprint arXiv:1202.3725 (2012).

[31] Aloke Guha. 2008. Method and system for proactive drive replacement for high availability storage systems. US Patent 7,373,559.

[32] Greg Hamerly, Charles Elkan, et al. 2001. Bayesian approaches to failure prediction for disk drives. In ICML, Vol. 1. 202-209.

[33] Milad Hashemi, Kevin Swersky, Jamie A Smith, Grant Ayers, Heiner Litz, Jichuan Chang, Christos Kozyrakis, and Parthasarathy Ranganathan. 2018. Learning memory access patterns. arXiv preprint arXiv:1803.02329 (2018).

[34] Mohamad H Hassoun et al. 1995. Fundamentals of artificial neural networks. MIT press.

[35] Kevin J Johnson and Robert E Synovec. 2002. Pattern recognition of jet fuels: comprehensive GC $\times$ GC with ANOVA-based feature selection and principal component analysis. Chemometrics and Intelligent Laboratory Systems 60, 1-2 (2002), 225-237.

[36] Myoungsoo Jung and Mahmut Kandemir. 2013. Revisiting widely held SSD expectations and rethinking system-level implications. In $A C M$ SIGMETRICS Performance Evaluation Review, Vol. 41. ACM, 203-216.

[37] Tae-Sung Jung, Young-Joon Choi, Kang-Deog Suh, Byung-Hoon Suh, Jin-Ki Kim, Young-Ho Lim, Yong-Nam Koh, Jong-Wook Park, Ki-Jong Lee, Jung-Hoon Park, et al. 1996. A 3.3 V 128 Mb multi-level NAND flash memory for mass storage applications. In 1996 IEEE International Solid-State Circuits Conference. Digest of TEchnical Papers, ISSCC. IEEE, $32-33$. 
[38] Dawon Kahng and Simon M Sze. 1967. A floating gate and its application to memory devices. The Bell System Technical fournal 46, 6 (1967), 1288-1295.

[39] Kenji Kira and Larry A Rendell. 1992. A practical approach to feature selection. In Machine Learning Proceedings 1992. Elsevier, 249-256.

[40] Kenji Kira, Larry A Rendell, et al. 1992. The feature selection problem: Traditional methods and a new algorithm. In Aaai, Vol. 2. 129-134.

[41] B Ravi Kiran, Dilip Mathew Thomas, and Ranjith Parakkal. 2018. An overview of deep learning based methods for unsupervised and semisupervised anomaly detection in videos. Journal of Imaging 4, 2 (2018), 36.

[42] Ana Klimovic, Heiner Litz, and Christos Kozyrakis. 2018. Selecta: Heterogeneous cloud storage configuration for data analytics. In 2018 \{USENIX\} Annual Technical Conference ( $\{$ USENIX $\}\{A T C\}$ 18). 759773.

[43] Daphne Koller and Mehran Sahami. 1996. Toward optimal feature selection. Technical Report. Stanford InfoLab.

[44] Cheolhyeon Kwon, Weiyi Liu, and Inseok Hwang. 2013. Security analysis for cyber-physical systems against stealthy deception attacks. In 2013 American control conference. IEEE, 3344-3349.

[45] Thomas Navin Lal, Olivier Chapelle, Jason Weston, and André Elisseeff. 2006. Embedded methods. In Feature extraction. Springer, 137-165.

[46] Andy Liaw, Matthew Wiener, et al. 2002. Classification and regression by randomForest. $R$ news 2,3 (2002), 18-22.

[47] M. Lindenbaum, S. Markovich, D. Rusakov, et al. 1999. Selective sampling for nearest neighbor classifiers. In Proceedings of The National Conference on Artificial Intelligence. 366-371.

[48] Geert Litjens, Thijs Kooi, Babak Ehteshami Bejnordi, Arnaud Arindra Adiyoso Setio, Francesco Ciompi, Mohsen Ghafoorian, Jeroen Awm Van Der Laak, Bram Van Ginneken, and Clara I Sánchez. 2017. A survey on deep learning in medical image analysis. Medical image analysis 42 (2017), 60-88.

[49] Heiner Litz and Milad Hashemi. 2020. Machine Learning for Systems. IEEE Micro 40, 5 (2020), 6-7.

[50] Fei Tony Liu, Kai Ming Ting, and Zhi-Hua Zhou. 2008. Isolation forest. In 2008 Eighth IEEE International Conference on Data Mining. IEEE, 413-422.

[51] Gilles Louppe, Louis Wehenkel, Antonio Sutera, and Pierre Geurts. 2013. Understanding variable importances in forests of randomized trees. In Advances in neural information processing systems. 431-439.

[52] Yixin Luo, Saugata Ghose, Yu Cai, Erich F Haratsch, and Onur Mutlu. 2018. Improving 3D NAND flash memory lifetime by tolerating early retention loss and process variation. Proceedings of the ACM on Measurement and Analysis of Computing Systems 2, 3 (2018), 37.

[53] Ao Ma, Rachel Traylor, Fred Douglis, Mark Chamness, Guanlin Lu, Darren Sawyer, Surendar Chandra, and Windsor Hsu. 2015. RAIDShield: characterizing, monitoring, and proactively protecting against disk failures. ACM Transactions on Storage (TOS) 11, 4 (2015), 17.

[54] Farzaneh Mahdisoltani, Ioan Stefanovici, and Bianca Schroeder. 2017. Improving storage system reliability with proactive error prediction. In Proceedings of the 2017 USENIX Conference on Usenix Annual Technical Conference. USENIX Association, 391-402.

[55] Farzaneh Mahdisoltani, Ioan Stefanovici, and Bianca Schroeder. 2017. Proactive error prediction to improve storage system reliability. In $2017\{$ USENIX\} Annual Technical Conference (\{USENIX $\}\{A T C\}$ 17). 391-402.

[56] Mary L McHugh. 2013. The chi-square test of independence. Biochemia medica: Biochemia medica 23, 2 (2013), 143-149.

[57] Justin Meza, Qiang Wu, Sanjev Kumar, and Onur Mutlu. 2015. A largescale study of flash memory failures in the field. In ACM SIGMETRICS Performance Evaluation Review, Vol. 43. ACM, 177-190.
[58] Mehdi Mohammadi, Ala Al-Fuqaha, Sameh Sorour, and Mohsen Guizani. 2018. Deep learning for IoT big data and streaming analytics: A survey. IEEE Communications Surveys \& Tutorials 20, 4 (2018), 2923-2960.

[59] Joseph F Murray, Gordon F Hughes, and Kenneth Kreutz-Delgado. 2005. Machine learning methods for predicting failures in hard drives: A multiple-instance application. fournal of Machine Learning Research 6, May (2005), 783-816.

[60] AW Pearson. 1972. The use of ranking formulae in R \& D projects. R\&D Management 2, 2 (1972), 69-73.

[61] Marco AF Pimentel, David A Clifton, Lei Clifton, and Lionel Tarassenko. 2014. A review of novelty detection. Signal Processing 99 (2014), 215249.

[62] Eduardo Pinheiro, Wolf-Dietrich Weber, and Luiz André Barroso. 2007. Failure trends in a large disk drive population. (2007).

[63] Apapan Pumsirirat and Liu Yan. 2018. Credit card fraud detection using deep learning based on auto-encoder and restricted boltzmann machine. International fournal of advanced computer science and applications 9, 1 (2018), 18-25.

[64] Cory Reche, Lee Nevill, and Tim Martin. 2012. Error detection/correction based memory management. US Patent 8,312,349.

[65] Maheshkumar Sabhnani and Gürsel Serpen. 2003. Application of Machine Learning Algorithms to KDD Intrusion Detection Dataset within Misuse Detection Context.. In MLMTA. 209-215.

[66] Mohammad Sabokrou, Mahmood Fathy, and Mojtaba Hoseini. 2016. Video anomaly detection and localisation based on the sparsity and reconstruction error of auto-encoder. Electronics Letters 52, 13 (2016), $1122-1124$

[67] Noelia Sánchez-Maroño, Amparo Alonso-Betanzos, and María Tombilla-Sanromán. 2007. Filter methods for feature selection-a comparative study. In International Conference on Intelligent Data Engineering and Automated Learning. Springer, 178-187.

[68] Bianca Schroeder and Garth A Gibson. 2007. Disk failures in the real world: What does an MTTF of 1, 000, 000 hours mean to you? In FAST, Vol. 7. 1-16.

[69] Bianca Schroeder, Raghav Lagisetty, and Arif Merchant. 2016. Flash reliability in production: The expected and the unexpected. In 14th $\{$ USENIX\} Conference on File and Storage Technologies ( $\{F A S T\} 16)$. $67-80$.

[70] Bianca Schroeder, Arif Merchant, and Raghav Lagisetty. 2017. Reliability of NAND-based SSDs: What field studies tell us. Proc. IEEE 105, 9 (2017), 1751-1769.

[71] Luis Talavera. 2005. An evaluation of filter and wrapper methods for feature selection in categorical clustering. In International Symposium on Intelligent Data Analysis. Springer, 440-451.

[72] Yongmin Tan, Hiep Nguyen, Zhiming Shen, Xiaohui Gu, Chitra Venkatramani, and Deepak Rajan. 2012. Prepare: Predictive performance anomaly prevention for virtualized cloud systems. In 2012 IEEE 32nd International Conference on Distributed Computing Systems. IEEE, 285294.

[73] Wei Wang, Yan Huang, Yizhou Wang, and Liang Wang. 2014. Generalized autoencoder: A neural network framework for dimensionality reduction. In Proceedings of the IEEE conference on computer vision and pattern recognition workshops. 490-497.

[74] Erci Xu, Mai Zheng, Feng Qin, Yikang Xu, and Jiesheng Wu. 2019. Lessons and actions: What we learned from $10 \mathrm{k}$ ssd-related storage system failures. In 2019 \{USENIX\} Annual Technical Conference ( $\{$ USENIX $\}\{A T C\}$ 19). 961-976.

[75] Yong Xu, Kaixin Sui, Randolph Yao, Hongyu Zhang, Qingwei Lin, Yingnong Dang, Peng Li, Keceng Jiang, Wenchi Zhang, Jian-Guang Lou, et al. 2018. Improving service availability of cloud systems by predicting disk error. In 2018 \{USENIX\} Annual Technical Conference 
( $\{$ USENIX $\}\{A T C\}$ 18). 481-494.

[76] Ji Hyuck Yun, Jin Hyuk Yoon, Eyee Hyun Nam, and Sang Lyul Min. 2012. An abstract fault model for NAND flash memory. IEEE Embedded Systems Letters 4, 4 (2012), 86-89.

[77] Jerrold H Zar. 2005. Spearman rank correlation. Encyclopedia of Biostatistics 7 (2005).

[78] Qiao Zhang, Guo Yu, Chuanxiong Guo, Yingnong Dang, Nick Swanson, Xinsheng Yang, Randolph Yao, Murali Chintalapati, Arvind Krishnamurthy, and Thomas Anderson. 2018. Deepview: Virtual disk failure diagnosis and pattern detection for azure. In 15th \{USENIX\} Symposium on Networked Systems Design and Implementation ( $\{$ NSDI\} 18). 519-532.

[79] Xu Zhang, Qingwei Lin, Yong Xu, Si Qin, Hongyu Zhang, Bo Qiao, Yingnong Dang, Xinsheng Yang, Qian Cheng, Murali Chintalapati, et al.
2019. Cross-dataset time series anomaly detection for cloud systems. In 2019 \{USENIX\} Annual Technical Conference (\{USENIX\} $\{$ ATC $\} 19)$. 1063-1076.

[80] Zijun Zhang. 2018. Improved Adam optimizer for deep neural networks. In 2018 IEEE/ACM 26th International Symposium on Quality of Service (IWQoS). IEEE, 1-2.

[81] Yang Zhou, Rong Jin, and Steven Chu-Hong Hoi. 2010. Exclusive lasso for multi-task feature selection. In Proceedings of the Thirteenth International Conference on Artificial Intelligence and Statistics. 988995.

[82] Hui Zou and Trevor Hastie. 2005. Regularization and variable selection via the elastic net. Fournal of the royal statistical society: series $B$ (statistical methodology) 67, 2 (2005), 301-320. 